\title{
Review of the monthly "Skyscape
}

\section{Archaeology Keynote Lecture Series" organised by the Journal of Skyscape Archaeology and the Sophia Centre, University of Wales Trinity Saint David, October 2020-March 2021}

\section{William F. Romain}

Indiana University, Museum of Archaeology and Anthropology wromain@iu.edu

The COVID-19 pandemic has made life difficult over the past year. There was one recurring bright spot, however: from October 2020 to March 2021 we were treated to a series of online presentations co-organised by the Sophia Centre at the University of Wales Trinity Saint David and the Journal of Skyscape Archaeology. There were 12 one-hour lectures in the series, which were hosted in turn by Nicholas Campion, Liz Henty and Kim Malville. The Zoom format allowed for live interaction and questions at the end of each presentation. Overall, the sessions were well-attended with 30-80 viewers for each session.

The presentations, by prominent researchers in the field, were exceptional. A major highlight in the last session was the announcement of the Lionel Sims Award for a student contribution that bridges the gap between anthropological theory and skyscape archaeology together with two Student Research Grants. Lionel Sims is one of the world's leading cultural astronomers, and he has long been an advocate for precisely the approach that this award encourages. To receive the Lionel Sims Award will be a high honour indeed!

The series got off to a great start with Carolyn Kennett's presentation "Insights into the Skyscape at The Hurlers Stone Circle Complex, Cornwall: Mapping the Sun and Reading the Hurlers". Situated in southwest Britain, The Hurlers is a Bronze Age complex 
that includes three stone circles, two standing stone monuments called The Pipers and a stone pavement that extends between the north and middle circles. During the course of two projects, solar and stellar alignment possibilities were assessed. Perhaps the most intriguing - and indeed compelling - alignment involves the Milky Way. The stone pavement that links two of the circles is comprised of multi-coloured stones, including quartz. Many of the stones are embedded in yellow clay soil. This suggested to the investigators that perhaps the pavement was intended to represent the Milky Way. Specifically, it was suggested that the trajectory of the Milky Way at spring equinox may have held special significance - as something of a pathway for the dead. For those who wish to know more about the archaeoastronomy of this site, a wonderful write-up was recently published in the Journal of Skyscape Archaeology (Nowakowski et al. 2020).

The second presentation of the day was by Alejandro López, titled "Through the Eyes of the Living: The Study of Present Societies and the Future of Cultural Astronomy". López has spent a considerable time working in the field as an ethnoastronomer, so he was able to cover many theoretical and methodological matters. He reminded us, for example, that as archaeoastronomers, our efforts to understand past societal beliefs are in large measure influenced by our own cultural understandings. As a consequence, what we present is our perspective about someone else's worldview. Obviously, this introduces biases. As archaeoastronomers we need to be aware of our preconceived notions or assumptions. Among the ways we can further that objective is by learning more about different aspects of anthropological and ethnographic theory and, when possible, engaging in fieldwork among indigenous peoples, or at the very least increase our reading of the ethnographic literature. Among the many useful recommendations that López offered was to keep in mind that that past ways of knowing about the sky were not static, but always evolving. And, whatever our ideas about the system we are studying, our ideas are like maps, "more simple than reality, [...] always incomplete and provisional".

During the question-and-answer session Liz Henty made reference to an important matter that López had noted in print, but that archaeoastronomers sometimes forget. We have a tendency to believe that certain practices or understandings are universal. López has explained why this is wrong:

An example of this is the importance of accuracy in astronomical measurements. In Western scientific astronomy, the accuracy of measurements is of key importance. This leads many researchers to view precision as a central concept in all astronomies. However, when it comes to the astronomical conceptions of many other cultures, the 'fuzziness' of measurements is in fact both necessary and desirable. (López 2011, 42)

This point alone should convince us that ethnoastronomy and the anthropology of religion offer useful perspectives in connection with our studies.

The first lecture for November 2020 was by Juan Belmonte, and was titled "From Giza to Petra: Land- and Skyscapes of the Ancient Middle East". Belmonte is one of the lead investigators for the OAS (Orientatio Ad Sidera) Project, a Spanish research team that has conducted archaeoastronomical work in a wide range of countries. For this series Belmonte gave us a tour of several sites where he and his team have worked. We began 
with Gobekli Tepe. Among the many celestial possibilities offered by the site is the clear orientation of one structure to the cardinal directions. As he pointed out, this may be the world's oldest cardinally aligned building. Moreover, a standing stone on the east side of the structure has the carved image of a lion, perhaps representing the constellation Leo at the time of equinox. Additionally, stellar alignments have been posited for other nearby structures. As Belmonte is fond of pointing out, however, Gobekli Tepe is an example of "testis unus, testis nullus" ("one witness is no witness"). In other words, although interpretations of stellar alignments and constellations represented by animal carvings at the site are intriguing, corroborating evidence is needed.

Our next stop was Egypt, where Belmonte and his team have investigated 400 sites. Among their findings is that sites were indeed oriented with respect to the Nile River, but also in many cases simultaneously oriented to the cardinal directions, or alternatively to the winter solstice. Space limitations preclude me from noting all the amazing discoveries that Juan told us about. For Egypt, however, I highly recommend Belmonte's co-edited volume, In Search of Cosmic Order: Selected Essays on Egyptian Archaeoastronomy (Belmonte and Shaltout 2009).

Next considered were ancient sites in Anatolia associated with the Hittites, where 61 structures including 40 temples were statistically assessed, finding evidence for solstitial alignments as well as orientations to east (possibly equinox). Moving south to the Nemrud Dag site in Turkey, Juan and his associates discovered solstitial alignments and a probable stellar alignment (to Regulus) in the design of the two main terraces at the site, likely associated with the coronation of King Antiochus I Theos. There was also an intriguing stone monument depicting a lion and, likely, the constellation Leo and a planetary conjunction.

Petra, the capital of the Nabataean Kingdom, was the last area discussed. Here the team identified a significant number of celestial alignments. Among the most notable is a winter solstice sunrise hierophany associated with the Lion Triclinium and a winter solstice sunset light-and-shadow effect on the inner sanctum of Ad Deir. Carved out of solid rock, Ad Deir is the structure that most people associate with Petra. Among the most important of the discoveries made at Petra was documentation of an equinox sunset alignment visible from the Al Madras structures in alignment with the summit of the sacred mountain, Jabal Haroun (for more on the Petra equinox phenomena see Belmonte et al. 2020).

In these discussions Belmonte was able to show how in each culture, the landscape, monumental sites, beliefs and rituals were intimately connected to the sky. We look forward to more amazing discoveries in the coming years.

The next presenter was Katya Stroud, with "A Neolithic World View Lost in Translation: Understanding the Megalithic Temples of Malta". The megalithic sites on Malta are among the oldest in the world, dating to the fourth and third millennia BC. Her presentation reminded us how interpretations of monumental structures are subject to change and often reflect the subjective views and preoccupations of scholars current at the time. Although one might think that archaeological narratives develop as a direct result of archaeological data, the reality is that other factors also influence our understandings. 
Among these factors, as she has previously written, are "pre-existing interpretations, the personal and social backgrounds of the excavator, the influence of contemporary national and international discoveries and the socio-political context of the discovery and interpretation" (Stroud 2019, 203).

To illustrate her point in the lecture, Stroud drew on the interpretive history of several temples, showing how, depending on the times, interpretations ranged from Giants having created the structures to preconceived notions of religious temples with special areas for priests and other areas for animal sacrifices. Another interpretation reflective of the times was that worship of a Mother Goddess was practised as reflected by female figurines found at the sites. Her conclusion was that today, interpretations are more about landscape and skyscape relationships - all of which begs the question of to what extent our interpretations are reflective of the ancient past or alternatively of our own subjective realities.

The lectures for December 2020 began with Kenneth Brophy's presentation “Megalithic Overkill and Knights on a Chessboard: Rescuing the Multiple Stone Rows of Northern Scotland from Alexander Thom". As the title implies, the several sites considered by Kenny are comprised of multiple rows of upright stones placed in long linear lines seemingly radiating out in a fan shape from a centre area. The stones are typically less than one metre in height. Alexander Thom is well-known for his research early in the history of archaeoastronomy. As Brophy explained, Thom believed that the stone rows were Bronze Age lunar observatories laid out using standard units of measurement, and that the rows could be used to predict lunar eclipses. However, archaeoastronomers have long recognised methodological problems in Thom's work, particularly related to his claims for high-precision lunar alignments. A priori assumptions and the selective use of data are just two of the difficulties that Brophy pointed out: another specific problem relates to the idealised grids that Thom drew for the stone rows. The declinations for the Moon at its extremes do not exactly match its rising and setting positions on the horizon. To deal with this, Thom proposed that the stone rows allowed an observer to "extrapolate" lunar azimuths. The problem is that Thom's grids showing where stones should be located do not correspond to what is found on the ground. To account for these discrepancies Thom claimed that the miscreant stones had moved as the result of soil creep.

In 2003 Brophy led a team that excavated at Battle Moss to test Thom's creeping stones idea. Excavation found that the stones they investigated had been set into dug holes and held in place by smaller stones packed on the sides. Therefore it is not possible for soil creep to have moved the stones. In hindsight it is evident that Thom's interpretation of the stone rows as lunar observatories is flawed. Indeed, to paraphrase Brophy, "these monuments could not have functioned in the way Thom suggested". Still, the stone rows of Scotland insist on explanation. And so what I found compelling was Brophy's response to a question from the audience - namely, what does he believe was the function of the stone rows? He suggested that although there may be celestial alignments not yet identified, rather than being tied to astronomic phenomena, perhaps they were related to the ancestors. Noting that the rows seem to "develop" relative to burials, perhaps individual stones were placed when a community member died. The 
result would be a "stone genealogy" whereby people could walk along a row and recall an ancestor memorialised by each stone - going back in time, toward the beginning of the family group. Brophy admits that this idea is "not provable", but he also suggests that this interpretation is congruent with understanding of the monuments as part of living communities rather than explanations that rely on obscure, complicated and arcane relationships to the Moon.

The next speaker was Marc Frincu, representing an international team that included Raul Perez-Enriquez, Levon Aghikyan and Simina Frincu. Marc presented on "Revisiting the Armenian Highlands: New Discoveries and Alternative Theories", and he covered four sites, starting with Carahunge. Sometimes called the "Armenian Stonehenge", Carahunge is comprised of a circle of stone menhirs surrounding a central cairn, with two so-called "arms" made up of dozens of stones extending in both a northern and southern direction from the centre circle. Various astronomical hypotheses had been suggested for this site, though none is convincing. Alternatively, Frincu showed how the stones comprising the north and south arms visually mimic the outline of mountains in the distance. The correspondences are quite remarkable. As he explained, walking along the arms gives one the feeling of being part of, or integrated with, the landscape.

The next site was Murad, which is characterised by petroglyphs, grave sites, cairns, rock enclosures called sheepfolds and modern graves. Analyses by the team identified possible solstice alignments perhaps serving a calendric function for sheep-herding peoples who have occupied the area for millennia. The third site was the Sevsar petroglyph site. The focus of the investigation was a large petroglyph involving a series of concentric ellipses surrounding a spiral design. At the centre of the petroglyph is a "cuphole" capable of supporting a wooden pole. Frincu suggested that the petroglyph could have been used as gnomon, with the shadows cast at the solstices and equinoxes captured by the design configuration (for more details about this compelling idea see Frincu et al. 2020).

The last site considered was Harthashen. It consists of two long stone rows, each made up of three parallel lines of stones. One row extends across the valley where the site is located; the other extends along the slope of an adjacent ridge. The two rows meet, thereby forming an apex. Several large cairns are associated with the structures. Hypotheses include an alignment to Arcturus in ca. 3000 BC, and a "framing" of the circumpolar circle. The circumpolar circle is the "maximum distance from the North Pole at which the stars are permanently above the horizon". In other words, the opening established by the $\mathrm{V}$-shape of the stone rows defines the parameters of the circumpolar area - a region of the sky considered by many cultures as the dwelling place of gods.

The first presentation for 2021 was given by Georg Zotti and was titled "Stellarium for Cultural Astronomy Research". I think it is fair to say that no other computer planetarium program has had as much impact on archaeoastronomy as Stellarium. Zotti was the developer of the ArchaeoLines Plug-in for Stellarium. This amazing tool plots the trajectories for the Sun, Moon, planets and stars for any date and time required.

Taking Stellarium to the next level, Zotti explained how the user can import landscape panoramas into the program. Thus, it is possible to recreate a view of the earth 
and sky from any site of interest, for the date and time selected. For those not able to watch this presentation, the methods for creating and loading landscape panoramas into Stellarium are explained in Chapter 7 of the "Stellarium 0.20.0 User Guide", which can be downloaded free of charge from the Stellarium website (http://stellarium.org/). Zotti next showed how 3D models and shadow effects can be incorporated into Stellarium analyses. Among the images shown were 3D simulations of the Sun at Stonehenge and the "serpent" shadow at El Castillo, Chichén Itza. It was almost as good as being there!

Stanislaw Iwaniszewski followed with "Relational Fields in Archaeoastronomy: The Mt Tlaloc Temple in Central Mexico". Tlaloc was one of the most important of the Mesoamerican gods, best known as the Aztec rain god. As implied by the term "relational field" in the title, Iwaniszewski's presentation revealed the many interrelated ways that Tlaloc was, and continues to be, important to human communities. The web of relationships begins with the topography of central Mexico and how that establishes the seasonal pattern of rains. The kinds of precipitation and fate of the crops depend on Tlaloc. Not only that, but the shape of agricultural fields reflects the site's quadripartite nature. And of considerable interest, important planting and harvesting dates in the agricultural season were based on a 260-day calendar, according to which the dates of alignments between ritual sites and Tlaloc mountain peaks were established. In the Aztec world there was a sophisticated relationship between the human community and the skyscape, the mountains, agricultural activity and the rains as provided by Tlaloc. Iwaniszewski's presentation reminded us that when looking at ancient sites we need a holistic approach. Everything is connected.

The first presentation in February was by Edwin C. Krupp, titled "Uplifted and Transported: Encounters at Burro Flats, California". Burro Flats Painted Cave is one of several Chumash/Tongva rock art sites in southern California which feature exquisite cave paintings. Krupp showed how, at the solstices, light interacts with certain of these paintings interactions he believes had spiritual significance for the people who periodically visited them. He also introduced us to a nearby site where the summer solstice sunrise causes a very distinct shadow effect on a bedrock boulder modified with shallow mortars, thus commemorating that event. The several photographs showing solstice light-and-shadow events as they interacted with cave paintings and landscape was fascinating. Beyond that, however, Krupp also explained that the importance of Burro Flats and other rock art is that it represents "the most archaic and fundamental symbolic vocabulary there is [...] and further, that symbolic vocabulary gives us entry into ancient belief systems".

Perhaps the most intriguing aspect of Krupp's talk was his juxtapositioning of the Burro Flats site with a modern-day research facility located just over a nearby ridge. Burro Flats is situated on the grounds of the Rocketdyne Santa Susana Field Laboratory. It was here that the Saturn V rocket and Space Shuttle engines were tested. As he pointed out, there is no place on the planet quite like Burro Flats. It is the "only place on earth where our modern world heritage in space converges with the prehistoric reach for the sky". It is his hope that Burro Flats will be inducted as a World Heritage site in recognition of its importance to the history of space exploration and Native American history.

Working in high mountain environments can be challenging. Yet that is exactly what Steven Gullberg did as he conducted extensive archaeoastronomical research in 
the Andes Mountains of Peru. His presentation, titled "Astronomy of the Inca Empire", highlighted some of his findings. He began by explaining how the Sun was of central importance to the Inca. Indeed, the Inca emperor was believed to be a direct descendant of the Sun. Solstices marked special festival dates and zenith passage dates were noted. Also of importance was the Milky Way, which was believed to be a celestial river. Gullberg explained how at the solstices the Sun and the Milky Way rise in the same region of the horizon, dividing the year into four quarters and thereby marking significant times of the year. He also showed us how various animals were seen in the dark areas of the Milky Way.

After this brief introduction Gullberg presented several highlights from his research. Having explored 31 sites, there were many to choose from. At Kenko Grande we witnessed summer solstice light-and-shadow effects creating the face of a puma and illuminating carved features in an associated cave. At the caves of Lacco, he showed how the northeast cave has an opening that directs light from the summer solstice sunrise onto an inner altar. And he showed how in the southeast cave, a light tube directs zenith sunlight to illuminate the inner altar. Moving on to Hacca 44 we learned how both solstice and equinox alignments are incorporated in the design of that site. At the famous Sacred Valley Region, he explained how the Q'espiwanka Pillars incorporate multiple solstice alignments. And - no small feat - Gullberg climbed to the summit of Cerro Unoraqui at $4733 \mathrm{~m}$ as well as Cerro Pumahuachana to investigate solstice alignments between Inca sites located on those peaks.

Of course, no assessment of Incan archaeoastronomy would be complete without Machu Picchu. Here Gullberg documented how the Sacred Plaza and Principal Temple are oriented to the summer solstice sunrise and winter solstice sunset. There are also solstitial, anti-zenith sunrise and Pleiades alignments, variously associated with the Intimachay Cave, Temple of the Condor, the Torreon, Wayna Pikchu and Gran Caverna. Certainly, among the most striking of all the phenomena that he showed us was the solstice sunrise alignment viewed from the Llactapata Sun Temple across to the Sacred Plaza of Machu Picchu, at a distance of about $5 \mathrm{~km}$. In a brief review such as this it is not possible to fully acknowledge all the interesting findings documented by his research. Fortunately, though, all the sites discussed in his presentation and many more are included in his new book Astronomy of the Inca Empire: Use and Significance of the Sun and the Night Sky (Gullberg 2020). This presentation was a nice introduction to his amazing work.

The lectures for March 2021 began with Duane Hamacher sharing some of what he has learned about indigenous Australian sky knowledge. The subject is of importance because, as he pointed out, the Aboriginal peoples have unbroken traditions going back more than 65,000 years. Thus, First Nations people in Australia are in possession of humankind's oldest knowledge about the sky. He explained that in Aboriginal Australia, sky knowledge informs people about all aspects of life, and he touched on a few of these stories as related to the Southern Cross, Moon and Milky Way. Especially interesting was his discussion of a prominent dark space in the Milky Way seen as an emu - the largest bird in Australia. Traditions about the Milky Way emu (and other animals) convey information about the seasons, timing of rainfall and the availability of food sources as well as informing people relative to social law, kinship and ceremony. 
As a source of traditional knowledge Aboriginal stories are important to preserve. As Hamacher rightly pointed out, however,

our connection to the stars, and the foundations of these ancient knowledge systems, are under constant threat. The advent of rapidly growing satellite networks overlapping the rapidly decreasing numbers of stars we can see due to light pollution is actively erasing this connection.

Hamacher sums up the situation quite well when he says that "preserving the visibility of the cosmos is at the core of the human experience and is critical for our future survival". Among the solutions that he mentioned is moving to amber LED lighting, better directed outdoor lighting and the creation of Dark Sky reserves (to learn more about Duane's work and indigenous Australian astronomy the reader is encouraged to explore the Australian Indigenous Astronomy website: www.aboriginalastronomy.com.au).

The last lecture of the series was Frank Prendergast's presentation, titled "Light and Shadows in Antiquity: Inferring Meaning Expressed in Past Materiality and Practices". He began by telling us about the historic development of several key concepts in the study of light and shadow. After that he presented some of the findings resulting from his 230 surveys of Neolithic passage tombs in Ireland. Analyses showed an orientation of several tombs to the solstices or equinoxes. The point that Prendergast emphasised, however, is that the deposition of human remains into the tombs involved movement from the realm of life and light and into dark chambers associated with death. For example, the Cairn H Carrowkeel tomb is aligned to the summer solstice sunset and, as he explained, the implication is that there is a connecting line of sorts extending from the dead in the tomb, out through the liminal entrance and along the solstice sightline to the liminal horizon between heaven and earth, where all celestial bodies set, including a deified Sun. Indeed, as he explained, "if archaeology is about anything these days it is about connecting sites like this to the ancestral dead". Moreover, what appears to have been important were not precision alignments, but rather, embedded symbolism. Other sites and phenomena that Prendergast illustrated included the equinox illumination of the inner burial chamber of the Cairn T Loughcrew passage tomb and the gnomon-kerb stone shadow effects at the Knowth passage tomb.

Certainly, the highlight of Prendergast's presentation for many of us was his discussion of the Newgrange passage tomb. For this site he showed us remarkable 3D graphics and photos illustrating the winter solstice sunrise hierophany wherein beams of sunlight penetrate deep into the tomb, illuminating the inner sanctum. Equally fascinating was his documentation of how, at winter solstice sunrise, large monoliths situated east of the tomb cast shadows onto the triple spiral motifs carved in several large target stones near the entrance. For the GC1-K1 phenomenon, the shadow track matches the diagonal trajectory of the spirals.

Prendergast's presentation brought this year's series to a conclusion on a high note. May the discoveries continue - aided, in part, by the Lionel Sims Award and student grants. 


\section{References}

Belmonte, J. A., A. C. González-García, A. Rodríguez-Antón and M. A. Perera Betancor, 2020. “Equinox in Petra: Land- and Skyscape in the Nabataean Capital". Nexus Network Journal 22: 369-391. https://doi. org/10.1007/s00004-019-00464-1

Belmonte, J. A. and M. Shaltout, eds., 2009. In Search of Cosmic Order: Selected Essays on Egyptian Archaeoastronomy. Cairo: Supreme Council of Antiquities Press.

Frincu, M. E., R. Perez-Enriquez and L. Aghikyan, 2020. "Revisiting Sevsar: Towards a Possible Gnomon in the Armenian Highlands". Journal of Skyscape Archaeology 6 (2): 207-220. https://doi.org/10.1558/jsa.19630

Gullberg, S., 2020. Astronomy of the Inca Empire: Use and Significance of the Sun and the Night Sky. Cham, Switzerland: Springer. https://doi.org/10.1007/978-3-030-48366-1

López, A. M., 2011. "Ethnoastronomy as an Academic Field: A Framework for a South American Program". In Archaeoastronomy and Ethnoastronomy: Building Bridges between Cultures: Proceedings of the 278th Symposium of the International Astronomical Union and "Oxford IX" International Symposium on Archaeoastronomy, Held in Lima, Peru, January 5-14, 2011, edited by C. L. N. Ruggles, 38-49. Cambridge: Cambridge University Press. https://doi.org/10.1017/S1743921311012452

Nowakowski, J. A., C. Kennett, J. Gossip and B. Sheen, 2020. "Investigating Archaeology and Astronomy at The Hurlers, Cornwall 2013-2019". Journal of Skyscape Archaeology 6 (1): 53-5. https://doi.org/10.1558/ jsa.39523

Stroud, K., 2019. "A Neolithic World View Lost in Translation: The Case of the Tarxien Temples". Journal of Skyscape Archaeology 5 (2): 191-209. https://doi.org/10.1558/jsa.40026 